\title{
Exposição ocupacional e ocorrência de pneumoconioses na região de Campinas (SP) Brasil, 1978-2003*
}

\author{
Occupational exposure and occurrence of pneumoconioses \\ in Campinas, Brazil, 1978-2003
}

\author{
Alessandro Vito Lido르, Satoshi Kitamura ${ }^{2}$, José Inácio Oliveira ${ }^{2}$, Sérgio Roberto de Lucca ${ }^{2}$, \\ Valmir Antonio Zulian de Azevedo ${ }^{2}$, Ericson Bagatin ${ }^{2}$
}

\begin{abstract}
Resumo
Objetivo: Desenvolver e consolidar uma ampla base de dados acerca da ocorrência das pneumoconioses numa região industrializada do Brasil, com especial referência às atividades mais freqüentemente relacionadas a essas doenças. Métodos: Numa avaliação retrospectiva observacional, coletaram-se dados referentes à casuística ambulatorial das pneumoconioses no Hospital das Clínicas da Universidade Estadual de Campinas, entre o período de 1978 e 2003. Incluíram-se os indivíduos com diagnóstico de pneumoconiose, com base no histórico ocupacional e no radiograma do tórax, segundo recomendações da Organização Internacional do Trabalho, de 1980 e 2000 , com anormalidades compatíveis com comprometimento intersticial do parênquima pulmonar. Resultados: Foram identificados 1.147 casos de pneumoconiose ( 1.075 homens e 72 mulheres), sendo 1.061 casos $(92,5 \%)$ de silicose, $51(4,45 \%)$ de pneumoconiose por poeira mista, $15(1,31 \%)$ de asbestose, $13(1,13 \%)$ de pneumoconiose por rocha fosfática e $7(0,61 \%)$ de outras pneumoconioses (por carvão, grafite e metais duros). As alterações radiológicas com profusão 1/0,1/1 e 1/2 e as pequenas opacidades regulares $p$, q e $r$ foram as mais freqüentes, tendo sido identificados 192 casos (16,74\%) com grandes opacidades. Observou-se redução pronunciada da ocorrência dos casos a partir da década de 1990; adicionalmente, o tempo de exposição foi caracteristicamente mais breve do que o observado em série norte-americana. Conclusões: Os dados do presente estudo estabelecem uma ampla base de dados para a investigação da ocorrência de pneumoconioses numa região industrializada brasileira, tornando factível a realização de estudos de seguimento e a elaboração de políticas de saúde relacionadas aos agravos respiratórios ocupacionais.
\end{abstract}

Descritores: Medicina do trabalho; Doenças respiratórias/epidemiologia; Pneumoconiose; Epidemiologia.

\begin{abstract}
Objective: To develop and consolidate a comprehensive database on the occurrence of pneumoconioses in an industrialized region of Brazil, with a special focus on the activities most frequently related to these diseases. Methods: A retrospective, observational study was conducted in order to gather data on cases of pneumoconioses treated at the outpatient clinic of the State University at Campinas Hospital das Clinicas between 1978 and 2003. Individuals diagnosed with pneumoconiosis, based on their occupational history and on chest X-ray findings of abnormalities consistent with interstitial lung disease involving the parenchyma, in accordance with the 1980 and 2000 recommendations of the International Labour Organization, were included in the study. Results: A total of 1147 cases of pneumoconiosis were identified (1075 in males and 72 in females): 1061 cases of silicosis (92.5\%); 51 cases of mixed-dust pneumoconiosis (4.45\%); 15 cases of asbestosis (1.31\%); 13 cases of phosphate rock-related pneumoconiosis (1.13\%); and 7 cases of other types of pneumoconiosis (0.6\%), including those related to exposure to coal, graphite and hard metals. The most common chest $X$-ray findings were $1 / 0,1 / 1$ or $1 / 2$ profusion and small regular opacities (p, q or r), although 192 patients (16.74\%) presented large opacities. There has been a substantial decline in the occurrence of the disease since the 1990s, and the duration of exposure was typically shorter than that observed in a study conducted in the United States. Conclusions: Our findings have been compiled into a comprehensive database for the investigation of pneumoconiosis in an industrialized area of Brazil. These data make it possible to conduct follow-up studies and develop health policies related to occupational respiratory disorders.
\end{abstract}

Keywords: Occupational medicine; Respiratory tract diseases/epidemiology; Pneumoconiosis; Epidemiology.

\footnotetext{
* Trabalho realizado na Área de Saúde do Trabalhador do Departamento de Medicina Preventiva e Social da Faculdade de Ciências Médicas da Universidade Estadual de Campinas - DMPS/FCM-Unicamp - Campinas (SP) Brasil.

1. Doutorando do Departamento de Medicina Preventiva e Social da Faculdade de Ciências Médicas da Universidade Estadual de Campinas - DMPS/FCM-Unicamp Campinas (SP) Brasil.

2. Professor Assistente Doutor da Área de Saúde do Trabalhador. Departamento de Medicina Preventiva e Social da Faculdade de Ciências Médicas da Universidade Estadual de Campinas - DMPS/FCM-Unicamp - Campinas (SP) Brasil.

Endereço para correspondência: Alessandro Vito Lido. Rua Vital Brasil, 100, Prédio do Cipoi, 3 Piso, Campus Universitário, Barão Geraldo, CEP 13083-888, Campinas, SP, Brasil.

Tel 5519 3521-7885/3521-8018. E-mail: lido@fcm.unicamp.br

Recebido para publicação em 22/3/2007. Aprovado, após revisão, em 15/8/2007.
} 


\section{Introdução}

A descrição da inalação de poeira nos ambientes de trabalho como agente causador de sintomas respiratórios e doenças pulmonares foi feita por Hipócrates, em 460 a.C., que observou essas alterações na saúde de mineradores. ${ }^{(1)} 0$ termo pneumoconiose, proposto por Zenker em 1866, caracteriza as alterações pulmonares parenquimatosas conseqüentes à inalação de poeira. ${ }^{(1)}$ Similarmente, na quarta Conferência Internacional de Pneumoconiose, realizada em Bucareste, em 1971, definiu-se pneumoconiose como 'uma reação tecidual pulmonar decorrente do acúmulo de poeira nos pulmões'.(2)

Milhares de novos casos são diagnosticados a cada ano em todo o mundo, com predominância nos países desenvolvidos, por terem um grande número de trabalhadores expostos em atividades de risco e em virtude das ações dos programas de vigilância à saúde ocupacional que registram e analisam a totalidade dos casos diagnosticados. No entanto, as pneumoconioses vêm apresentando um acentuado declínio nesses países, nos quais as estatísticas correlatas são realizadas por órgãos especializados na coleta, registro e processamento desses dados, que são utilizados na vigilância e no controle das doenças ocupacionais. ${ }^{(3-5)}$

No Brasil, entretanto, poucos dados estão disponíveis acerca da ocorrência das pneumoconioses, bem como das características das exposições ocupacionais relacionadas, por se tratar de doenças subdiagnosticadas e subnotificadas pelas empresas e pelos profissionais de saúde e em virtude da falta de amplos estudos epidemiológicos e de programas de vigilância à saúde dos trabalhadores expostos aos seus agentes causadores. ${ }^{(6)}$ Portanto, este estudo justifica-se pela tentativa de consolidação e estruturação de informações sistematizadas a partir da demanda ambulatorial dos pacientes diagnosticados com pneumoconiose, em um serviço terciário de referência na região de abrangência do Hospital das Clínicas da Universidade Estadual de Campinas (HC-Unicamp), uma área densamente povoada e com um grande parque industrial. Tais dados permitiriam conhecer o número de pacientes diagnosticados nesse serviço, constituindo uma base de dados original para a realização de outros estudos e para a elaboração de políticas de saúde.

\section{Métodos}

Trata-se de um estudo retrospectivo observacional de pacientes diagnosticados com pneumoconioses, nos ambulatórios de Medicina do Trabalho e Pneumologia do HC-Unicamp e do registro dos trabalhadores identificados com pneumoconiose no banco de dados da Área de Saúde do Trabalhador da Faculdade de Ciências Médicas (FCM)-Unicamp proveniente dos inquéritos epidemiológicos realizados no período de 1978 a 2003. Os critérios estabelecidos para o diagnóstico das pneumoconioses, neste estudo, seguem as recomendações estabelecidas pela International Labour Organization (ILO, Organização Internacional do Trabalho): história clínica e ocupacional compatíveis e radiograma do tórax com profusão de pequenas opacidades no parênquima pulmonar maior ou igual a $1 / 0 .{ }^{(7)} 0$ protocolo deste estudo foi aprovado pelo Comitê de Ética em Pesquisa da FCM-Unicamp.

Para o estabelecimento do histórico ocupacional, obtiveram-se todos os antecedentes laborais, com descrição do ramo de atividade das empresas, ocupações desempenhadas pelo paciente com o tempo de trabalho em cada atividade, bem como a descrição dos agentes químicos, físicos e biológicos a que estiveram expostos nesses ambientes de trabalho. Essas informações foram obtidas através da anamnese ocupacional realizada por médicos (residentes e pós-graduandos) supervisionados por docentes experientes na sua realização. As informações prestadas pelos pacientes também eram verificadas através da análise dos registros em carteira profissional.

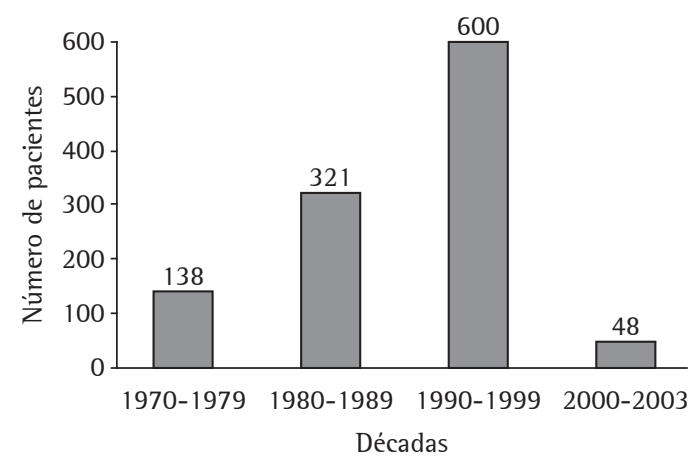

Figura 1 - Pneumoconioses: distribuição do diagnóstico, por décadas, dos pacientes do Hospital das Clínicas da Universidade Estadual de Campinas. 
Quanto ao tabagismo, os pacientes foram separados em fumantes e não-fumantes. 0 consumo de cigarro foi quantificado em anos/maço; consideraram-se fumantes todos os pacientes que tinham um histórico de tabagismo de pelo menos um cigarro por dia, por mais de um ano, e os que continuavam fumando.

Os radiogramas do tórax foram interpretados, conjuntamente, por dois ou mais leitores, com experiência específica de interpretação, de acordo com os critérios da Classificação Internacional das Radiografias das Pneumoconioses da 1LO, segundo as revisões de 1980 e $2000 .{ }^{(7)}$ No caso de discordância, um terceiro leitor foi requisitado, para limitar a variabilidade de interpretações intra e interleitores. ${ }^{(8,9)}$ As informações obtidas foram registradas em formulário padrão, seguindo o modelo da ILO. Quando os exames radiológicos não foram localizados, consideramos os dados da leitura radiológica registrados em prontuários médicos, em formulário padrão, com técnica de interpretação padronizada pela ILO, de 1980 e 2000, com exames radiológicos de boa qualidade e interpretados por dois ou mais leitores experientes.

As alterações radiológicas foram classificadas em: forma simples, em que ocorrem pequenas opacidades radiológicas, com diâmetros inferiores a $10 \mathrm{~mm}$; e forma complicada, também conhecida como fibrose maciça progressiva, em que ocorrem grandes opacidades, com diâmetros maiores de $10 \mathrm{~mm}$, resultantes da coalescência das pequenas opacidades.

A partir das informações coletadas nos formulários padronizados, foi constituído um banco de dados, elaborado através do programa Microsoft Access, versão 2000. 0 estudo descritivo das informações foi sumarizado através de medidas de tendência central e dispersão (média e desvio padrão ou mediana e variação).

\section{Resultados}

Foram obtidos dados referentes a 1.147 pacientes, sendo $1.075(93,72 \%)$ do sexo masculino e $72(6,28 \%)$ do sexo feminino. A média de idade foi de $47 \pm 10$ anos, com mediana de 46 anos. 0 tempo médio de exposição aos agentes causadores das pneumoconioses foi de $17 \pm 8$ anos, e a mediana foi de 16 anos. Analisando a distribuição das pneumoconioses por faixa etária, 88 pacientes $(9,12 \%)$ foram diagnosticados até os 34 anos de idade, e $743(77 \%)$ com idade entre 35 e 59 anos. Quanto à procedência dos pacientes, identificamos 52 municípios, situados nos estados de São Paulo, Minas Gerais, Bahia e Paraná, compreendendo uma população de 5.273.358 habitantes, segundo o censo do Instituto Brasileiro de Geografia e Estatística de 2000.

Conforme observado na Figura 1, a maioria dos casos foram diagnosticados nas décadas de 1980 e 1990, com tendência à redução na década seguinte. Em relação à distribuição dos tempos de exposição, encontramos 55 casos $(4,8 \%)$ com tempo menor de 5 anos, $170(14,8 \%)$ entre 5 e 9 anos de exposição e 780 casos (68\%) com tempo superior a 9 anos. Desta forma, a maioria dos pacientes com pneumoconiose tiveram um tempo de exposição entre 10 e 24 anos, com apenas 6,5\% apresentando valores maiores do que 30 anos. Tais dados contrastam com os observados nos estados de Michigan, Nova Jersey e Ohio, nos Estados Unidos, ${ }^{(4)}$ onde apenas 7\% tinham tempo de exposição menor do que 10 anos, enquanto 32,3\% foram expostos por mais de 30 anos (Tabela 1). Na Figura 2, observa-se que o início da exposição, no nosso meio, concentrou-se nas décadas de 1950 a 1970, com redução pronunciada a partir dos anos 80 .

$\mathrm{Na}$ análise da qualidade dos radiogramas, encontramos, respectivamente, $623(54,3 \%)$ e 524 exames

Tabela 1 - Distribuição do tempo de exposição, em anos, dos pacientes com pneumoconiose atendidos no Hospital das Clínicas da Universidade Estadual de Campinas, de 1978 a 2003, e dos casos identificados nos Estados Unidos pelo National Institute for Occupational Safety and Health, de 1989 a 1999.

\begin{tabular}{lcc}
\hline & $\begin{array}{c}\text { Estados norte- } \\
\text { americanos: Michigan, } \\
\text { Nova Jersey e Ohio } \\
(1989 \text { a 1998) }\end{array}$ & $\begin{array}{c}\text { Pacientes da } \\
\text { Unicamp } \\
(1978 \text { a 2003) }\end{array}$ \\
\cline { 2 - 3 } & $\mathrm{n}(\%)$ & $\mathrm{n}(\%)$ \\
\hline Inferior a 10 anos & $83(7,0)$ & $225(19,6)$ \\
10 a 20 anos & $227(19,2)$ & $378(33,0)$ \\
21 a 30 anos & $324(27,5)$ & $328(28,6)$ \\
Superior a 30 anos & $381(32,3)$ & $74(6,5)$ \\
Desconhecido & $165(14,0)$ & $142(12,4)$ \\
Total & $1.180(100,0)$ & $1.147(100,0)$ \\
\hline
\end{tabular}

*Work-related lung disease surveillance report 2002 (Relatório de vigilância de doenças pulmonares ocupacionais), National Institute for Occupational Safety and Health, 2003.(2) 


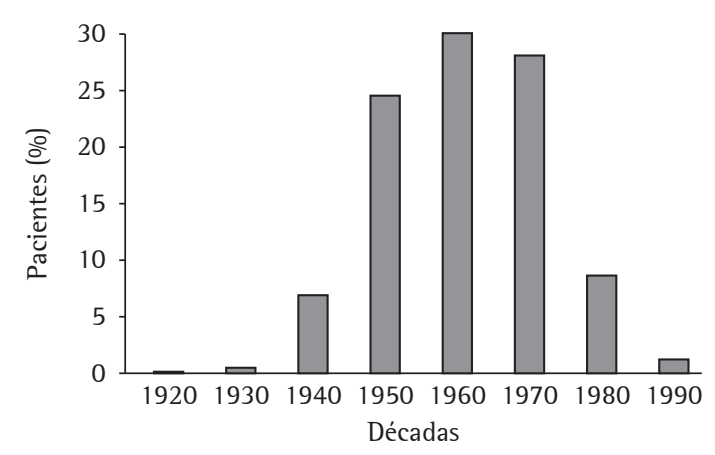

Figura 2 - Pneumoconioses: distribuição do início da exposição, por décadas, dos pacientes atendidos no Hospital das Clínicas da Universidade Estadual de Campinas.

$(45,7 \%)$ com qualidades 1 e 2 . Os radiogramas do tórax foram agrupados segundo a profusão predominante, nas categorias $0,1,2$ e 3 , da seguinte forma: em 1 , as profusões $1 / 0,1 / 1$ e $1 / 2$; em 2 , as profusões $2 / 1,2 / 2$ e $2 / 3$; e em 3 , as profusões $3 / 2$, $3 / 3$ e $3 /+$. As alterações radiológicas com profusão 1 e as pequenas opacidades regulares $p, q, r$ foram as mais freqüentes, tendo sido identificados 192 casos $(16,74 \%)$ com grandes opacidades (Tabela 2).

A Tabela 3 mostra a exposição ocupacional aos agentes causadores das pneumoconioses: a sílica livre cristalina correspondeu a 1.061 dos casos $(92,5 \%)$; a poeira mista, formada por corindo e carborundo, a 51 casos $(4,5 \%)$; o asbesto, a $15(1,3 \%)$; a rocha fosfática, a 13 (1,1\%); e outros agentes (carvão, grafite e metais), a 7 casos $(0,6 \%)$ (Tabela 3). 0 tempo médio de exposição aos agentes causadores das pneumoconioses, em anos, foi de 17,1 $\pm 8,4$, com mediana de 16, mínimo de 1 e máximo de 45.

Através da história ocupacional, identificamos a ocorrência de pneumoconiose em 21 tipos de atividades industriais. Estiveram expostos à sílica 821 pacientes $(77,67 \%)$ na indústria cerâmica, $17(1,6 \%)$ na produção de vidro, $11(1,04 \%)$ na produção de pedras decorativas e $50(4,7 \%)$ na indústria metalúrgica, nas operações de fundição de metais em caixas de areia, jateamento e esmerilhamento de peças. No entanto, encontramos 44 pacientes $(4,16 \%)$ com histórico de exposição à poeira mista, na produção de material abrasivo do tipo corindo e carborundo, $15(1,43 \%)$ expostos ao asbesto, na indústria de fibrocimento, freios e isolamento térmico industrial, e 3 pacientes
Tabela 2 - Classificação da qualidade dos radiogramas, da profusão, forma e tamanho das pequenas e grandes opacidades, de acordo com a Classificação Internacional das Radiografias das Pneumoconioses (revisão da Organização Internacional do Trabalho, de 1980 e 2000).

\begin{tabular}{|c|c|c|}
\hline & $n$ & $\%$ \\
\hline \multicolumn{3}{|l|}{ Profusão } \\
\hline 1 & 663 & 57,8 \\
\hline 2 & 339 & 29,6 \\
\hline 3 & 145 & 12,6 \\
\hline Total & 1.147 & 100,0 \\
\hline \multicolumn{3}{|c|}{ Forma/tamanho } \\
\hline $\mathrm{p} / \mathrm{p}$ & 295 & 26,2 \\
\hline$p / q$ & 221 & 19,6 \\
\hline $\mathrm{p} / \mathrm{r}$ & 3 & 0,3 \\
\hline Total & 519 & 46,1 \\
\hline$q / p$ & 21 & 1,9 \\
\hline$q / q$ & 299 & 26,6 \\
\hline$q / r$ & 105 & 9,3 \\
\hline Total & 425 & 37,8 \\
\hline$r / p$ & 0 & 0 \\
\hline$r / q$ & 7 & 0,6 \\
\hline$r / r$ & 117 & 10,4 \\
\hline Total & 124 & 11,1 \\
\hline$s / s$ & 8 & 0,7 \\
\hline$s / t$ & 13 & 1,2 \\
\hline $\mathrm{s} / \mathrm{u}$ & 0 & 0 \\
\hline Total & 21 & 1,9 \\
\hline$t / s$ & 0 & 0 \\
\hline$t / t$ & 23 & 2,0 \\
\hline $\mathrm{t} / \mathrm{u}$ & 11 & 0,1 \\
\hline Total & 34 & 3,0 \\
\hline $\mathrm{u} / \mathrm{s}$ & 0 & 0 \\
\hline $\mathrm{u} / \mathrm{t}$ & 1 & 0,1 \\
\hline $\mathrm{u} / \mathrm{u}$ & 1 & 0,1 \\
\hline Total & 2 & 0,2 \\
\hline \multicolumn{3}{|c|}{ Grande opacidade } \\
\hline$A$ & 58 & 5,0 \\
\hline B & 72 & 6,3 \\
\hline C & 62 & 5,4 \\
\hline total & 192 & 16,7 \\
\hline
\end{tabular}

expostos $(0,3 \%)$ à poeira de grafite, na produção de eletrodos. Na atividade de metalurgia, $2(0,2 \%)$ estiveram expostos à poeira metálica de rebolos especiais. Na mineração de superfície e de profundidade, incluindo as pedreiras, 52 pacientes (4,91\%) com pneumoconiose estiveram expostos à sílica, $2(0,2 \%)$ ao carvão mineral e $2(0,2 \%)$ à poeira mista. 
Tabela 3 - Distribuição dos agentes causadores das pneumoconioses e tempo de exposição na população em estudo.

\begin{tabular}{lcc}
\hline \multicolumn{1}{c}{ Agente } & $\mathrm{n}(\%)$ & $\begin{array}{c}\text { Tempo de exposição, } \\
\text { mediana (variação) }\end{array}$ \\
\hline Asbesto & $15(1,3)$ & $16,7(3,3-27,2)$ \\
Carvão mineral & $2(0,2)$ & $7,0(2,0-12,0)$ \\
Grafite & $3(0,3)$ & $18,3(16,0-20,5)$ \\
Poeira metálica & $2(0,2)$ & $21,7(13,0-30,4)$ \\
Poeira mista & $51(4,5)$ & $13,0(1,6-26,0)$ \\
Rocha fosfática & $13(1,1)$ & $7,0(2,5-16,7)$ \\
Sílica & $1061(92,5)$ & $20,6(1,0-53,0)$ \\
Total & $1147(100,0)$ & - \\
\hline
\end{tabular}

Encontramos 43 tipos de ocupações relacionados à ocorrência das pneumoconioses, e evidenciamos uma maior freqüência em: estampadores, que fazem trabalho manual de modelagem das peças de cerâmicas (248 casos; $29,42 \%)$; forneiros $(72 ; 8,54 \%)$ e esmaltadores $(69 ; 8,19 \%)$, que trabalham na atividade de preparo das peças cerâmicas nos fornos de curagem; e nos moleiros, operadores de moinho de trituração da matéria prima da indústria cerâmica (85; 10,08\%).

\section{Discussão}

O HC-Unicamp, situado no distrito de Barão Geraldo, no município de Campinas, estado de São Paulo, iniciou suas atividades ambulatoriais em 1978, para atendimento em nível terciário, através dos sistemas públicos de saúde. No mesmo período, com a implantação da Área de Saúde do Trabalhador, do Departamento de Medicina Preventiva e Social da FCM-Unicamp, criou-se o Ambulatório de Medicina do Trabalho. Desde então, tornou-se um centro de referência no atendimento dos trabalhadores, para o diagnóstico das doenças ocupacionais, ensino e pesquisa, na região de Campinas.

Entre os primeiros estudos sobre silicose realizados no Brasil, destacam-se os realizados no início da década de 1970 nos municípios de Pedreira e Jundiaí, no estado de São Paulo. ${ }^{(10,11)}$ Tais estudos demonstraram uma elevada ocorrência de silicose em ceramistas, investigando-a de forma mais abrangente a partir do diagnóstico de um caso nessa categoria profissional, que era considerada, naquela época, como pouco importante para a gênese da doença. Em 1995, ${ }^{(12)}$ publicou-se a ocorrência de 818 casos de silicose na região de Campinas, em diversos ramos da atividade industrial, e foi recomendada a criação de um programa de vigilância epidemiológica para melhor controle desses ambientes de trabalho. Poucos estudos investigaram com método epidemiológico uma população de trabalhadores expostos; em 1998, ${ }^{(13)}$ um estudo avaliou 4.279 trabalhadores empregados nas indústrias cerâmicas do município de Pedreira, diagnosticando 159 casos de silicose, com prevalência de 4,7\% do total de indivíduos expostos na localidade. Um estudo realizado na indústria do fibrocimento avaliou 828 ex-trabalhadores, identificando 74 casos (5,9\%) de asbestose e 246 casos $(29,7 \%)$ com alterações pleurais. Além disso, observou-se piora da função pulmonar nos casos de maior profusão radiológica. ${ }^{(14)}$ Sobre a atividade de mineração do amianto, há também um estudo transversal de morbidade entre 4.220 trabalhadores expostos ao asbesto, no período de 1940 a 1996. ${ }^{(15)}$ Com base nos dados da história clínica e ocupacional, nos exames da função pulmonar e nas alterações do radiograma do tórax e da tomografia computadorizada de alta resolução, foram observados 17 casos $(0,4 \%)$ com fibrose, 18 casos $(0,43 \%)$ de fibrose com placas e 71 casos $(1,68 \%)$ com placas pleurais. ${ }^{(15)}$

Embora tais estudos tenham trazido informações pontuais acerca da epidemiologia das pneumoconioses no Brasil, com as informações obtidas nesse estudo, procuramos estabelecer uma ampla base de dados para futuras investigações, correlacionando-as com as informações derivadas de outros inquéritos epidemiológicos. Um achado particularmente interessante do presente estudo foi a diferença do tempo de exposição em relação a uma amostra avaliada nos Estados Unidos (Tabela 1).(4) Nela, pode-se observar que o maior contingente de nossa população afetada foi exposto à sílica posteriormente ao ocorrido com a população norteamericana, provavelmente em virtude do atraso no desenvolvimento do parque industrial brasileiro. Portanto, observamos, em nosso estudo, que os pacientes tiveram um tempo de exposição menor do que o encontrado na população norte-americana, possivelmente em razão da maior concentração de sílica nos ambientes de trabalho. A menor ocorrência de silicose nas mulheres, 6,28\% dos casos diagnosticados neste estudo, está relacionada à pequena participação das mulheres nessas atividades de trabalho, principalmente nas décadas de 1950, 1960 e 1970, período considerado como crítico 
para a geração de doença, graças ao elevado nível de exposição a poeiras. A participação do trabalho feminino concentrava-se em atividades manuais e leves, de acabamento, avaliação de qualidade e embalagem da louça cerâmica.

Em relação ao ramo de atividade e à função, identificamos os ceramistas estampadores de louça doméstica e sanitária, seguidos pelos trabalhadores de fundições, empresas mineradoras e pedreiras, como as categorias profissionais de maior ocorrência da silicose, semelhante aos achados de um estudo realizado no estado norte-americano de Nova Jersey, ${ }^{(3)}$ e do Sentinel Event Notification System for Occupational Risks (Sistema de Notificação de Eventos-Sentinela para Riscos Ocupacionais) no período de 1989 a 1998, nos Estados Unidos. ${ }^{(4)}$

0 radiograma do tórax é considerado um dos instrumentos básicos para o diagnóstico da pneumoconiose, ao lado da história ocupacional. Em nossa casuística, destacamos a presença de 192 grandes opacidades $(16,74 \%)$, fibrose maciça progressiva classificadas como A em 58 casos (5,05\%), B em $72(6,28 \%)$ e C em $62(5,41 \%)$ - nos radiogramas do tórax dos pacientes com tempo médio de exposição de $17 \pm 8,4$ anos. Em 1989, ${ }^{(16)}$ realizou-se um estudo com 276 ceramistas com diagnóstico de silicose, no Reino Unido, com tempo médio de exposição de $35 \pm 10$ anos, o qual identificou a presença de grandes opacidades nos radiogramas de 115 indivíduos com silicose $(41,65 \%)$, sendo $67(24,3 \%)$ do tipo A, $44(15,9 \%)$ do tipo B e $4(1,45 \%)$ do tipo C. ${ }^{(16)}$ Essa grande diferença, em relação ao estudo atual, possivelmente se deve ao maior tempo de exposição ou às condições relacionadas ao período da exposição.

Em conclusão, os dados do presente estudo estabelecem uma ampla base de dados para a investigação da ocorrência de pneumoconioses numa região industrializada brasileira, tornando factível a realização de estudos de seguimento e a elaboração de políticas de saúde relacionadas aos agravos respiratórios ocupacionais.

\section{Referências}

1. Corn JK. Historical aspects of industrial hygiene--II. Silicosis. Am Ind Hyg Assoc J. 1980;41(2):125-33.

2. National Institute for Occupational Safety and Health. Health Effects of Occupational Exposure to Respirable Crystalline Silica. NIOSH hazard review. Cincinnati: Dept. of Health and Human Services, Centers for Disease Control and Prevention, National Institute for Occupational Safety and Health, 2002.

3. Rosenman KD, Reilly MJ, Henneberger PK Estimating the total number of newly-recognized silicosis cases in the United States. Am J Ind Med. 2003;44(2):141-7.

4. National Institute for Occupational Safety and Health. WorkRelated lung Disease Surveillance Report, 2002. Cincinnati: U.S. Dept. of Health and Human Services, Public Health Service, Centers for Disease Control and Prevention, 2003.

5. Finkelstein MM. Silicosis surveillance in Ontario from 1979 to 1992. Scand J Work Environ Health. 1995;21 Suppl 2:55-7.

6. Algranti E. Epidemiologia das doenças ocupacionais respiratórias no Brasil. In: Menezes AM. Epidemiologia das doenças respiratórias. Rio de Janeiro: Revier; 2001. p.119-43.

7. International Labour Office. Guidelines for the Use of ILO International Classification of Radiographs of Pneumoconiosis. Occupational Safety and Health Series n. 22 (rev). Genebra: ILO; 2000.

8. Valiante DJ, Rosenman KD. Does silicosis still occur? JAMA. 1989;262(21):3003-7.

9. Mulloy KB, Coultas DB, Samet JM. Use of chest radiographs in epidemiological investigations of pneumoconioses. $\mathrm{Br} \mathrm{J}$ Ind Med. 1993;50(3):273-5.

10. Morrone LC. Epidemiologia da silicose no estado de São Paulo. Rev Bras. Saúde Ocup. 1980;8(1):6-30.

11. Nogueira DP, Certain D, Brolio R, Garrafa NM, Shibata H. Ocorrência de silicose entre trabalhadores da indústria cerâmica da cidade de Jundiaí, SP (Brasil). Rev Saúde Pública. 1981;15(3):263-71.

12. Bagatin E, Jardim JR, Nery LE, Capitani EM, Marchi E, Sabino $\mathrm{MO}$, et al. Ocorrencia de silicose pulmonar na regiao de Campinas - SP. Jornal De Pneumologia. 1995;21(1):17-26.

13. Oliveira Jl. Prevalência da silicose entre os trabalhadores das indústrias cerâmicas do município de Pedreira (SP) [thesis]. Campinas: Universidade Estadual de Campinas; 1998.

14. Algranti E, Mendonça EM, DeCapitani EM, Freitas JB, Silva $\mathrm{HC}$, Bussacos MA. Non-malignant asbestos-related diseases in Brazilian asbestos-cement workers. Am J Ind Med. 2001; 40(3):240-54.

15. Bagatin E, Neder JA, Nery LE, Terra-Filho M, Kavakama J, Castelo A, et al. Non-malignant consequences of decreasing asbestos exposure in the Brazil chrysotile mines and mills. Occup Environ Med. 2005;62(6):381-9.

16. Prowse K, Allen MB, Bradbury SP. Respiratory symptoms and pulmonary impairment in male and female subjects with pottery workers' silicosis. Ann Occup Hyg. 1989;33(3): 375-85. 